\title{
The Multiple Summation Formula and Polylogarithms
}

\author{
Milorad R. Stevanović*
}

AbStRaCt. In this paper is given the formula:

$$
\begin{aligned}
& F_{n}(x) \equiv \sum_{k_{1}=1}^{\infty} \frac{x^{k_{1}}}{k_{1}} \sum_{k_{2}=1}^{k_{1}} \frac{x^{k_{2}}}{k_{2}} \cdots \sum_{k_{n}=1}^{k_{n-1}} \frac{x^{k_{n}}}{k_{n}}=\sum_{\substack{\sum_{j=1}^{n} j \cdot \alpha_{j}=n \\
\alpha_{j} \geq 0}} \frac{\prod_{k=1}^{n} \zeta_{k}^{\alpha_{k}}\left(x^{k}\right)}{\prod_{k=1}^{n} k^{\alpha_{k}} \alpha_{k} !} \\
& n \geq 1, \quad-1 \leq x<1
\end{aligned}
$$

with

$$
\zeta_{k}(x) \equiv L i_{k}(x) \equiv \sum_{r=1}^{\infty} \frac{x^{r}}{r^{k}} \quad(k \geq 0),
$$

and the method by which it can be obtained.

\section{INTRODUCTION}

First of all let us introduce the series with theirs notations:

$$
\zeta_{k}(x) \equiv L i_{k}(x) \equiv \sum_{r=1}^{\infty} \frac{x^{r}}{r^{k}} \quad(k \geq 0)
$$

It is evident that is $\zeta_{0}(x)=\frac{x}{1-x}, \quad \zeta_{1}(x)=-\log (1-x) \quad(-1 \leq x<1)$, and for $k \geq 2, \zeta_{k}(\mathrm{x})$ is represented by uniformly and absolutely convergent series in region $-1 \leq x \leq 1$. For these functions, known as polylogarithms and annotated by $L i_{k}(x)$, an old Legendre's notation will be used in this paper, which also was used by Ramanujan. For functions $F_{n}(x)$ we can take $F_{0}(x)=1$ and for $n=1$ we have $F_{1}(x)=\zeta_{1}(x)$.

Lemma 1.1. Series for $F_{n}(x)$ is convergent for $-1 \leq x<1 \quad(n \geq 1)$.

2000 Mathematics Subject Classification. Primary: 40B05; Secondary: 33E99.

Key words and phrases. multiple sums, multiple summation, polylogarithms.

${ }^{*}$ Research supported by Science Fund of Serbia under Grant 1457. 
Proof. For $|x| \leq\left|x_{0}\right|<1$ we have:

$$
\begin{aligned}
\left|F_{n}(x)\right| & \leq F_{n}(|x|) \leq F_{n}\left(\left|x_{0}\right|\right)<\sum_{k_{1}=1}^{\infty} \frac{\left|x_{0}\right|^{k_{1}}}{k_{1}}\left(\sum_{k_{2}=1}^{k_{1}} \frac{1}{k_{2}}\right)^{n-1}< \\
& <\sum_{k_{1} \geq k_{1}^{\prime}} \frac{\left|x_{0}\right|^{k_{1}}}{k_{1}} 2^{n-1} \log ^{n-1} k_{1}+A\left(k_{1}^{\prime}\right)< \\
& <2^{n-1} \sum_{k_{1} \geq k_{1}^{\prime \prime}}^{\left|x_{0}\right|^{k_{1}}}+A\left(k_{1}^{\prime}\right)+A\left(k_{1}^{\prime \prime}\right)<+\infty,
\end{aligned}
$$

where $A\left(k_{1}^{\prime}\right), A\left(k_{1}^{\prime \prime}\right) A\left(k_{1}^{\prime}\right), A\left(k_{1}^{\prime \prime}\right)$ are positive constants, and:

$$
\sum_{k_{2}=1}^{k_{1}} \frac{1}{k_{2}}<2 \log k_{1} \quad\left(k_{1} \geq k_{1}^{\prime}\right) \quad \text { and } \quad \frac{\log ^{n-1} k_{1}}{k_{1}}<1 \quad\left(k_{1} \geq k_{1}^{\prime \prime} \geq k_{1}^{\prime}\right)
$$

By Weierstrass criterion for uniform convergence we conclude that the power series for $F_{n}(x)$ is uniformly and absolutely convergent in one closed subdomain of $(-1,1)$. It has remained to be proved that $F_{n}(-1)$ exists. In order to prove it let us write:

$$
\begin{aligned}
(-1)^{n+1} F_{n+1}(-1) & =\sum_{k=1}^{\infty} \frac{(-1)^{k-1}}{k} \sum_{k_{1}}^{k} \frac{(-1)^{k_{1}-1}}{k_{1}} \cdots \sum_{k_{n}=1}^{k_{n-1}} \frac{(-1)^{k_{n}-1}}{k_{n}}= \\
& =\sum_{k=1}^{\infty} \frac{(-1)^{k-1}}{k} v(n, k)=\sum_{k=1}^{\infty}(-1)^{k-1} a_{k} .
\end{aligned}
$$

If we prove:

1. $\lim _{k \rightarrow \infty} a_{k}=0$,

2. $d_{t}^{(s)}=\frac{1}{2 t-1} v(s, 2 t-1)-\frac{1}{2 t} v(s, 2 t)>0 \Rightarrow a_{2 t-1}>a_{2 t} \quad(\forall t \in N)$,

3. $v(s, t)>0 \quad(s, t \in N) \quad \Rightarrow \quad a_{k}>0 \quad(\forall k \geq 1)$,

from Leibnitz criterion for alternatively series will follow the convergence of series:

$$
\sum_{k=1}^{\infty}(-1)^{k-1} a_{k}
$$

and the existence of $F_{n}(-1)$.

Indeed, we have: 
1.

$$
\begin{aligned}
& 0 \leq\left|\lim _{k \rightarrow \infty} a_{k}\right|=\lim _{k \rightarrow \infty} \frac{|v(n, k)|}{k} \leq \lim _{k \rightarrow \infty} \frac{1}{k}\left(\sum_{k_{1}=1}^{k} \frac{1}{k_{1}}\right)^{n-1} \leq \\
& \leq 2^{n-1} \lim _{k \rightarrow \infty} \frac{\log ^{n-1} k}{k}=0 \Rightarrow \\
& \lim _{k \rightarrow \infty} a_{k}=0 .
\end{aligned}
$$

2. Prove by induction for $s$.

$$
\begin{aligned}
& d_{t}^{(1)}=\frac{1}{(2 t-1) 2 t}\left\{\sum_{j=1}^{2 t} \frac{(-1)^{j-1}}{j}+1\right\}>0 \quad(\forall t) \\
& d_{t}^{(2)}=\frac{1}{(2 t-1) 2 t}\{v(2,2 t)+v(1,2 t-1)\} \\
& v(2,2 t)=\sum_{j=1}^{t} d_{j}^{(1)}>0, \quad v(1,2 t-1)>0 \Rightarrow d_{t}^{(2)}>0 \quad(\forall t), \\
& d_{t}^{(3)}=\frac{1}{(2 t-1) 2 t}\{v(3,2 t)+v(2,2 t-1)\} \\
& \left.\begin{array}{rl}
v(3,2 t) & =\sum_{j=1}^{t} d_{j}^{(2)}>0, \\
2,2 t-1) & =v(2,2 t)+\frac{1}{2 t} v(1,2 t)>0
\end{array}\right\} \Rightarrow d_{t}^{(3)}>0 \quad(\forall t), \\
& d_{t}^{(s+1)}=\frac{1}{(2 t-1) 2 t}\{v(s+1,2 t)+v(s, 2 t-1)\}, \\
& v(s+1,2 t)=\sum_{j=1}^{t} d_{j}^{(s)}>0, \quad v(s, 2 t-1)=v(s, 2 t)+\frac{1}{2 t} v(s-1,2 t) \\
& \Rightarrow \quad d_{t}^{(s+1)}=\frac{1}{(2 t-1) 2 t}\left\{\sum_{j=1}^{t} d_{j}^{(s)}+\sum_{j=1}^{t} d_{j}^{(s-1)}+\frac{1}{2 t} \sum_{j=1}^{t} d_{j}^{(s-2)}\right\} \quad(s \geq 3, \forall t)
\end{aligned}
$$

By assumption of induction, from here, we conclude that is $d_{t}^{(s+1)}>0$ and by induction 2 . is proved. 
3.

$$
\begin{aligned}
v(1, t)>0 \quad(\forall t), & \quad \text { (follows from 2.), } \\
v(2, t)>0 \quad(\forall t) & \quad(\text { follows from 2.) }, \\
v(s, 2 t) & =\sum_{j=1}^{t} d_{j}^{(s-1)}>0 \quad(s \geq 2) \quad(s \geq 2) .
\end{aligned}
$$

Thus, we have proved lemma 1.

\section{Lemma 1.2.}

$$
F_{r}^{\prime}(x)=\frac{1}{x} \sum_{j=0}^{r-1} \zeta_{j}\left(x^{j+1}\right) F_{r-1-j}(x) \quad(-1 \leq x<1),(r \geq 1) .
$$

Proof.

$$
\begin{aligned}
& F_{r}^{\prime}(x)=\sum_{k_{1}=1}^{\infty} x^{k_{1}-1} \sum_{k_{2}=1}^{k_{1}} \frac{x^{k_{2}}}{k_{2}} \cdots \sum_{k_{r}=1}^{k_{r-1}} \frac{x^{k_{r}}}{k_{r}}+ \\
& +\sum_{p=2}^{r-1} \sum_{k_{1}=1}^{\infty} \frac{x^{k_{1}}}{k_{1}} \cdots \sum_{k_{p}=1}^{k_{p-1}} x^{k_{p}-1} \cdots \sum_{k_{r}=1}^{k_{r-1}} \frac{x^{k_{r}}}{k_{r}}+ \\
& +\sum_{k_{1}=1}^{\infty} \frac{x^{k_{1}}}{k_{1}} \sum_{k_{2}=1}^{k_{1}} \frac{x^{k_{2}}}{k_{2}} \cdots \sum_{k_{r-1}=1}^{k_{r-2}} \frac{x^{k_{r-1}}}{k_{r-1}} \sum_{k_{r}=1}^{k_{r-1}} \frac{x^{k_{r}}}{k_{r}}= \\
& =S_{1}+\sum_{p=2}^{r-1} S_{p}+S_{r} \\
& S_{1}=\sum_{k_{2}=1}^{\infty} \frac{x^{k_{2}}}{k_{2}} \cdots \sum_{k_{r}=1}^{k_{r-1}} \frac{x^{k_{r}}}{k_{r}} \sum_{k_{1}=k_{2}}^{\infty} x^{k_{1}-1}= \\
& =\frac{1}{x(1-x)} \sum_{k_{2}=1}^{\infty} \frac{x^{2 k_{2}}}{k_{2}} \sum_{k_{3}=1}^{k_{2}} \frac{x^{k_{3}}}{k_{3}} \cdots \sum_{k_{r}=1}^{k_{r-1}} \frac{x^{k_{r}}}{k_{r}}= \\
& =\frac{1}{x(1-x)} \sum_{k_{1}=1}^{\infty} \frac{x^{2 k_{1}}}{k_{1}} \sum_{k_{2}=1}^{k_{1}} \frac{x^{k_{2}}}{k_{2}} \cdots \sum_{k_{r-1}=1}^{k_{r-2}} \frac{x^{k_{r-1}}}{k_{r-1}}= \\
& =\frac{1}{x(1-x)} T_{1} \text {. }
\end{aligned}
$$


Let $T_{p}$ be the series obtain from $F_{r-1}(x)$ substituting $x^{k_{p}}$ by $x^{2 k_{p}} \quad(1 \leq p \leq r)$.

$$
\begin{aligned}
S_{p} & =\sum_{k_{1}=1}^{\infty} \frac{x^{k_{1}}}{k_{1}} \cdots \sum_{k_{p-1}=1}^{k_{p-2}} \frac{x^{k_{p-1}}}{k} \sum_{p-1}^{k_{p-1}} \frac{x^{k_{p+1}}}{k_{p+1}=1} \cdots \sum_{k_{r}=1}^{k_{r-1}} \frac{x^{k_{r}}}{k_{r}} \sum_{k_{p}=k_{p+1}}^{k_{p-1}} x^{k_{p}-1}= \\
& =\frac{1}{x(1-x)} T_{p}-\frac{1}{1-x} T_{p-1} \quad(2 \leq p \leq r-1) .
\end{aligned}
$$

In the same way it follows that:

$$
\begin{aligned}
S_{r} & =\frac{1}{1-x} F_{r-1}(x)-\frac{1}{1-x} T_{r-1} \Rightarrow \\
\Rightarrow \quad F_{r}^{\prime}(x) & =\frac{1}{x(1-x)} T_{1}+ \\
& +\sum_{p=2}^{r-1}\left\{\frac{1}{x(1-x)} T_{p}-\frac{1}{1-x} T_{p-1}\right\}+ \\
& +\frac{1}{1-x} F_{r-1}(x)-\frac{1}{1-x} T_{r-1} \Rightarrow \\
\Rightarrow \quad F_{r}^{\prime}(x) & =\frac{1}{x} \sum_{p=1}^{r-1} T_{p}+\frac{1}{1-x} F_{r-1}(x) .
\end{aligned}
$$

Further,

$$
\begin{aligned}
T_{r-1} & =\sum_{k_{1}=1}^{\infty} \frac{x^{k_{1}}}{k_{1}} \sum_{k_{2}=1}^{k_{1}} \frac{x^{k_{2}}}{k_{2}} \cdots \sum_{k_{r-2}=1}^{k_{r-3}} \frac{x^{k_{r-2}}}{k_{r-2}} \sum_{k_{r-1}=1}^{k_{r-2}} \frac{x^{2 k_{r-1}}}{k_{r-1}}= \\
& =\sum_{k_{1}=1}^{\infty} \frac{x^{k_{1}}}{k_{1}} \sum_{k_{2}=1}^{k_{1}} \frac{x^{k_{2}}}{k_{2}} \cdots \sum_{k_{r-1}=1}^{k_{r-3}} \frac{x^{2 k_{r-1}}}{k_{r-1}} \sum_{k_{r-2}=k_{r-1}}^{k_{r-3}} \frac{x^{k_{r-2}}}{k_{r-2}}= \\
& =\sum_{k_{1}=1}^{\infty} \frac{x^{k_{1}}}{k_{1}} \sum_{k_{2}=1}^{k_{1}} \frac{x^{k_{2}}}{k_{2}} \cdots \sum_{k_{r-2}=1}^{k_{r-3}} \frac{x^{3 k_{r-2}}}{k_{r-2}^{2}}+ \\
& +\sum_{k_{1}=1}^{\infty} \frac{x^{k_{1}}}{k_{1}} \sum_{k_{2}=1}^{k_{1}} \frac{x^{k_{2}}}{k_{2}} \cdots \sum_{k_{r-2}=1}^{k_{r-3}} \frac{x^{2 k_{r-2}}}{k_{r-2}} \sum_{k_{r-1}=1}^{k_{r-3}} \frac{x^{k_{r-1}}}{k_{r-1}}-T_{r-2}= \\
= & \sum_{k_{1}=1}^{\infty} \frac{x^{k_{1}}}{k_{1}} \sum_{k_{2}=1}^{k_{1}} \frac{x^{k_{2}}}{k_{2}} \cdots \sum_{k_{r-2}=1}^{k_{r-4}} \frac{x^{3 k_{r-2}}}{k_{r-2}^{2}} \sum_{k_{r-3}=k_{r-2}}^{k_{r-4}} \frac{x^{k_{r-3}}}{k_{r-3}}+ \\
+ & \sum_{k_{1}=1}^{\infty} \frac{x^{k_{1}}}{k_{1}} \sum_{k_{2}=1}^{k_{1}} \frac{x^{k_{2}}}{k_{2}} \cdots \sum_{k_{r-4}}^{k_{r-2}=1} \frac{x^{2 k_{r-2}}}{k_{r-2}} \sum_{k_{r-3}=k_{r-2}}^{k_{r-4}} \frac{x^{k_{r-3}}}{k_{r-3}} \sum_{k_{r-1}=1}^{k_{r-3}} \frac{x^{k_{r-1}}}{k_{r-1}}-T_{r-2}=
\end{aligned}
$$




$$
\begin{aligned}
& =\sum_{k_{1}=1}^{\infty} \frac{x^{k_{1}}}{k_{1}} \sum_{k_{2}=1}^{k_{1}} \frac{x^{k_{2}}}{k_{2}} \cdots \sum_{k_{r-3}=1}^{k_{r-4}} \frac{x^{4 k_{r-3}}}{k_{r-3}^{3}}+ \\
& +\sum_{k_{1}=1}^{\infty} \frac{x^{k_{1}}}{k_{1}} \sum_{k_{2}=1}^{k_{1}} \frac{x^{k_{2}}}{k_{2}} \cdots \sum_{k_{r-3}=1}^{k_{r-4}} \frac{x^{3 k_{r-3}}}{k_{r-3}^{2}} \sum_{k_{r-2}=1}^{k_{r-4}} \frac{x^{k_{r-2}}}{k_{r-2}}+ \\
& +\sum_{k_{1}=1}^{\infty} \frac{x^{k_{1}}}{k_{1}} \sum_{k_{2}=1}^{k_{1}} \frac{x^{k_{2}}}{k_{2}} \cdots \sum_{k_{r-3}=1}^{k_{r-4}} \frac{x^{2 k_{r-3}}}{k_{r-3}} \sum_{k_{r-2}=1}^{k_{r-4}} \frac{x^{k_{r-2}}}{k_{r-2}} \sum_{k_{r-1}=1}^{k_{r-2}} \frac{x^{k_{r-1}}}{k_{r-1}}-T_{r-3}-T_{r-2}= \\
& =\sum_{k_{1}=1}^{\infty} \frac{x^{k_{1}}}{k_{1}} \sum_{k_{2}=1}^{k_{1}} \frac{x^{k_{2}}}{k_{2}} \cdots \sum_{k_{r-4}=1}^{k_{r-5}} \frac{x^{5 k_{r-4}}}{k_{r-4}^{4}}+ \\
& +\sum_{k_{1}=1}^{\infty} \frac{x^{k_{1}}}{k_{1}} \sum_{k_{2}=1}^{k_{1}} \frac{x^{k_{2}}}{k_{2}} \cdots \sum_{k_{r-4}=1}^{k_{r-5}} \frac{x^{4 k_{r-4}}}{k_{r-4}^{3}} \sum_{k_{r-3}=1}^{k_{r-5}} \frac{x^{k_{r-3}}}{k_{r-3}}+ \\
& +\sum_{k_{1}=1}^{\infty} \frac{x^{k_{1}}}{k_{1}} \sum_{k_{2}=1}^{k_{1}} \frac{x^{k_{2}}}{k_{2}} \cdots \sum_{k_{r-4}=1}^{k_{r-5}} \frac{x^{3 k_{r-4}}}{k_{r-4}^{2}} \sum_{k_{r-3}=1}^{k_{r-5}} \frac{x^{k_{r-3}}}{k_{r-3}} \sum_{k_{r-2}=1}^{k_{r-3}} \frac{x^{k_{r-2}}}{k_{r-2}}+ \\
& +\sum_{k_{1}=1}^{\infty} \frac{x^{k_{1}}}{k_{1}} \sum_{k_{2}=1}^{k_{1}} \frac{x^{k_{2}}}{k_{2}} \cdots \sum_{k_{r-2}=1}^{k_{r-3}} \frac{x^{3 k_{r-2}}}{k_{r-2}^{2}}+ \\
& +\sum_{k_{1}=1}^{\infty} \frac{x^{k_{1}}}{k_{1}} \sum_{k_{2}=1}^{k_{1}} \frac{x^{k_{2}}}{k_{2}} \cdots \sum_{k_{r-2}=1}^{k_{r-3}} \frac{x^{2 k_{r-2}}}{k_{r-2}} \sum_{k_{r-1}=1}^{k_{r-3}} \frac{x^{k_{r-1}}}{k_{r-1}}-T_{r-2}= \\
& =\sum_{k_{1}=1}^{\infty} \frac{x^{k_{1}}}{k_{1}} \sum_{k_{2}=1}^{k_{1}} \frac{x^{k_{2}}}{k_{2}} \cdots \sum_{k_{r-4}=1}^{k_{r-5}} \frac{x^{2 k_{r-4}}}{k_{r-4}} \sum_{k_{r-3}=1}^{k_{r-5}} \frac{x^{k_{r-3}}}{k_{r-3}} \sum_{k_{r-2}=1}^{k_{r-3}} \frac{x^{k_{r-2}}}{k_{r-2}} \sum_{k_{r-1}=1}^{k_{r-2}} \frac{x^{k_{r-1}}}{k_{r-1}}- \\
& -T_{r-4}-T_{r-3}-T_{r-2}=\sum_{k_{1}=1}^{\infty} \frac{x^{k_{1}}}{k_{1}} \sum_{k_{2}=1}^{k_{1}} \frac{x^{2 k_{2}}}{k_{2}} \sum_{k_{3}=1}^{k_{1}} \frac{x^{k_{3}}}{k_{3}} \cdots \sum_{k_{r-1}=1}^{k_{r-2}} \frac{x^{k_{r-1}}}{k_{r-1}}+ \\
& +\sum_{k_{1}=1}^{\infty} \frac{x^{k_{1}}}{k_{1}} \sum_{k_{2}=1}^{k_{1}} \frac{x^{3 k_{2}}}{k_{2}^{2}} \sum_{k_{3}=1}^{k_{1}} \frac{x^{k_{3}}}{k_{3}} \cdots \sum_{k_{r-2}=1}^{k_{r-3}} \frac{x^{k_{r-2}}}{k_{r-2}}+\sum_{k_{1}=1}^{\infty} \frac{x^{k_{1}}}{k_{1}} \sum_{k_{2}=1}^{k_{1}} \frac{x^{4 k_{2}}}{k_{2}^{3}} \sum_{k_{3}=1}^{k_{1}} \frac{x^{k_{3}}}{k_{3}} \cdots \\
& \cdots \sum_{k_{r-3}=1}^{k_{r-4}} \frac{x^{k_{r-3}}}{k_{r-3}}+\cdots+\sum_{k_{1}=1}^{\infty} \frac{x^{k_{1}}}{k_{1}} \sum_{k_{2}=1}^{k_{1}} \frac{x^{(r-1) k_{2}}}{k_{2}^{r-2}}-\sum_{j=2}^{r-2} T_{j}=
\end{aligned}
$$




$$
\begin{aligned}
& -\sum_{j=2}^{r-2} T_{j}+\left(\sum_{k_{2}=1}^{\infty} \frac{x^{3 k_{2}}}{k_{2}^{2}} \sum_{k_{3}=1}^{k_{2}} \frac{x^{k_{3}}}{k_{3}} \cdots \sum_{k_{r-1}=1}^{k_{r-2}} \frac{x^{k_{r-1}}}{k_{r-1}}+\zeta_{1}\left(x^{2}\right) F_{r-2}(x)-T_{1}\right)+ \\
& +\left(\sum_{k_{2}=1}^{\infty} \frac{x^{4 k_{2}}}{k_{2}^{3}} \sum_{k_{3}=1}^{k_{2}} \frac{x^{k_{3}}}{k_{3}} \cdots \sum_{k_{r-2}=1}^{k_{r-3}} \frac{x^{k_{r-2}}}{k_{r-2}}+\zeta_{2}\left(x^{3}\right) F_{r-3}(x)-\right. \\
& \left.-\sum_{k_{2}=1}^{\infty} \frac{x^{3 k_{2}}}{k_{2}^{2}} \sum_{k_{3}=1}^{k_{2}} \frac{x^{k_{3}}}{k_{3}} \cdots \sum_{k_{r-1}=1}^{k_{r-2}} \frac{x^{k_{r-1}}}{k_{r-1}}\right)+\left(\sum_{k_{2}=1}^{\infty} \frac{x^{5 k_{2}}}{k_{2}^{4}} \sum_{k_{3}=1}^{k_{2}} \frac{x^{k_{3}}}{k_{3}} \cdots\right. \\
& \left.\ldots \sum_{k_{r-3}} \frac{x^{k_{r-3}}}{k_{r-3}}+\zeta_{3}\left(x^{4}\right) F_{r-4}(x)-\sum_{k_{2}=1}^{\infty} \frac{x^{4 k_{2}}}{k_{2}^{3}} \sum_{k_{3}=1}^{k_{2}} \frac{x^{k_{3}}}{k_{3}} \cdots \sum_{k_{r-2}=1}^{k_{r-3}} \frac{x^{k_{r-2}}}{k_{r-2}}\right)+\cdots+ \\
& +\left(\zeta_{r-1}\left(x^{r}\right)+\zeta_{r-2}\left(x^{r-1}\right) F_{1}(x)-\sum_{k_{2}=1}^{\infty} \frac{x^{(r-1) k_{2}}}{k_{2}^{r-2}} \sum_{k_{3}=1}^{k_{2}} \frac{x^{k_{3}}}{k_{3}}\right)= \\
& =-\sum_{j=1}^{r-2} T_{j}+\sum_{j=1}^{r-1} \zeta_{j}\left(x^{j+1}\right) F_{r-1-j}(x) \Rightarrow
\end{aligned}
$$

$$
T_{r-1}=-\sum_{j=1}^{r-2} T_{j}+\sum_{j=1}^{r-1} \zeta_{j}\left(x^{j+1}\right) F_{r-1-j}(x) .
$$

From (3) and (4) with $\zeta_{0}(x)=\frac{x}{1-x}$ follows formula (2).

\section{Lemma 1.3.}

$$
n F_{n}(x)=\sum_{j=0}^{n-1} \zeta_{n-j}\left(x^{n-j}\right) F_{j}(x) \quad(-1 \leq x<1) \quad(n \geq 1) .
$$

Proof. Formula (5) can be proved by induction. For $n=1$ it is true because of $F_{0}(x)=1, \quad F_{1}(x)=\zeta_{1}(x)$.

$$
\begin{aligned}
\left(\sum_{j=0}^{n} \zeta_{n+1-j}\left(x^{n+1-j}\right) F_{j}(x)\right)^{\prime} & =\frac{1}{x} \sum_{j=0}^{n}(n+1-j) \zeta_{n-j}\left(x^{n+1-j}\right) F_{j}(x)+ \\
& +\sum_{j=0}^{n} \zeta_{n+1-j}\left(x^{n+1-j}\right) F_{j}^{\prime}(x)
\end{aligned}
$$

since

$$
\left(\zeta_{k}\left(x^{k}\right)\right)_{x}^{\prime}=\frac{k}{x} \zeta_{k-1}\left(x^{k}\right)
$$


Second sum on the right-hand side of (6) is:

$$
\begin{aligned}
& \sum_{j=0}^{n} \zeta_{n+1-j}\left(x^{n+1-j}\right) F_{j}^{\prime}(x) \stackrel{(2)}{=} \\
= & \frac{1}{x} \sum_{j=1}^{n} \zeta_{n+1-j}\left(x^{n+1-j}\right) \sum_{s=0}^{j-1} \zeta_{s}\left(x^{s+1}\right) F_{j-1-s}(x)= \\
= & \frac{1}{x} \sum_{s=0}^{n-1} \zeta_{s}\left(x^{s+1}\right) \sum_{j=s+1}^{n} \zeta_{n+1-j}\left(x^{n+1-j}\right) F_{j-1-s}(x)= \\
= & \frac{1}{x} \sum_{s=0}^{n-1} \zeta_{s}\left(x^{s+1}\right) \sum_{t=0}^{(n-s)-1} \zeta_{(n-s)-t}\left(x^{(n-s)-t}\right) F_{t}(x) \stackrel{(*)}{=} \\
= & \frac{1}{x} \sum_{s=0}^{n-1}(n-s) \zeta_{s}\left(x^{s+1}\right) F_{n-s}(x)=\frac{1}{x} \sum_{j=0}^{n} j \zeta_{n-j}\left(x^{n+1-j}\right) F_{j}(x) .
\end{aligned}
$$

Sign $(*)$ denotes that we applied assumption of induction that formula (5) is true for every $1 \leq k \leq n$.

From (6) and (8) we get:

$$
\begin{aligned}
\left(\sum_{j=0}^{n} \zeta_{n+1-j}\left(x^{n+1-j}\right) F_{j}(x)\right)^{\prime} & =\frac{n+1}{x} \sum_{j=0}^{n} \zeta_{n-j}\left(x^{n+1-j}\right) F_{j}(x) \stackrel{(2)}{=} \\
& =\left((n+1) F_{n+1}(x)\right)^{\prime} \Rightarrow \\
\Rightarrow(n+1) F_{n+1}(x) & =\sum_{j=0}^{n} \zeta_{n+1-j}\left(x^{n+1-j}\right) F_{j}(x)+C
\end{aligned}
$$

and for $x=0$ we have $C=0$, so the formula (5) is true for $n+1$, and by induction it is true for every natural $n$.

Theorem 1.1.

$$
\begin{array}{r}
F_{n}(x) \equiv \sum_{k_{1}=1}^{\infty} \frac{x^{k_{1}}}{k_{1}} \sum_{k_{2}=1}^{k_{1}} \frac{x^{k_{2}}}{k_{2}} \cdots \sum_{k_{n}=1}^{k_{n-1}} \frac{x^{k_{n}}}{k_{n}}=\sum_{\substack{\sum_{j=1}^{n} j \cdot \alpha_{j}=n \\
\alpha_{j} \geq 0}} \frac{\prod_{k=1}^{n} \zeta_{k}^{\alpha_{k}}\left(x^{k}\right)}{\prod_{k=1}^{n}\left(k^{\alpha_{k}} \alpha_{k} !\right)} \\
(n \geq 1), \quad(-1 \leq x<1) .
\end{array}
$$

Proof. Formula (9) follows from (5) and from assumption of induction that formula (9) is true for every $1 \leq k \leq n-1$. In sum $\frac{1}{n} \sum_{j=1}^{n} \zeta_{j}\left(x^{j}\right) F_{n-j}(x)$, for some combination $\sum_{j=1}^{n} j\left(\alpha_{j}\right)_{0}=n$, in numerators for $j=1,2, \ldots, n$ appear respectively 
(some of them is obtained from the previous ones) $j\left(\alpha_{j}\right)_{0}$, which in sum gives:

$$
\frac{1}{n} \sum_{j=1}^{n} j\left(\alpha_{j}\right)_{0} \frac{\prod_{k=1}^{n} \zeta_{k}^{\left(\alpha_{k}\right)_{0}}\left(x^{k}\right)}{\prod_{k=1}^{n}\left(k^{\left(\alpha_{k}\right)_{0}}\left(\alpha_{k}\right)_{0} !\right)},
$$

which at the end leads to the formula (9) for $n$.

Corollary 1.1. Using the formulas:

$$
\zeta_{k}(-1)=-\left(1-\frac{1}{2^{k-1}}\right) \zeta_{k}(1) \quad(k \geq 2), \quad \zeta_{1}(-1)=-\log 2,
$$

with $\zeta_{k}=\zeta(k), \zeta_{k}(-1)=\zeta(-k)$, from (9) we get:

$$
\begin{aligned}
F_{3}(-1)= & -\frac{1}{6} \log ^{3} 2-\frac{1}{2} \zeta(2) \log 2-\frac{1}{4} \zeta(3), \\
F_{4}(-1)= & -\frac{1}{24} \log ^{4} 2+\frac{1}{4} \zeta(2) \log ^{2} 2+\frac{1}{4} \zeta(3) \log 2+\frac{9}{16} \zeta(4), \\
F_{5}(-1)= & -\frac{1}{120} \log ^{5} 2-\frac{1}{12} \zeta(2) \log ^{3} 2-\frac{1}{8} \zeta(3) \log ^{2} 2- \\
& -\frac{9}{16} \zeta(4) \log 2-\frac{1}{8} \zeta(3) \zeta(2)-\frac{3}{16} \zeta(5) .
\end{aligned}
$$

Corollary 1.2. From recurrence relation (5) it follows:

$$
\exp \left(\sum_{j=1}^{\infty} \frac{\zeta_{j}\left(x^{j}\right)}{j} t^{j}\right)=\sum_{n=0}^{\infty} F_{n}(x) t^{n} \quad(-1 \leq x<1) .
$$

\section{REFERENCES}

[1] A.M. Legendre, Traité des Fonctions Elliptiques et des Intégrales Eulériennes, Vol. 2., Paris: Huzard-Courcier, 1826.

[2] N. Nielsen, Handbuch der theorie der Gammafunktion, Leipzig: Teubner, 1906; Reprint: New York: Chelsea, 1965.

[3] S. Ramanujan, Notebooks, Bombay: Tata Institute of Fundamental Research, 1957.

Technical Faculty,

Svetog Save 65,

32000 C̆AČAK

Serbia and Montenegro

E-mail address: milmath@tfc.kg.ac.yu 